\title{
Targeting Cellular Metabolism Chemosensitizes the Doxorubicin-Resistant Human Breast Adenocarcinoma Cells
}

\author{
Shulan Ma, ${ }^{1}$ Rongfei Jia, ${ }^{2}$ Dongju Li, ${ }^{1}$ and Bo Shen ${ }^{3}$ \\ ${ }^{1}$ Training Center of Medical Experiments, Basic Medical School, Fudan University, Shanghai 200032, China \\ ${ }^{2}$ Department of Oncology, Shanghai Xuhui Central Hospital, Shanghai 200031, China \\ ${ }^{3}$ Institute of Radiation Medicine, Fudan University, Shanghai 200032, China \\ Correspondence should be addressed to Bo Shen; shenbo@fudan.edu.cn
}

Received 27 June 2015; Revised 25 September 2015; Accepted 29 September 2015

Academic Editor: Siyaram Pandey

Copyright (C) 2015 Shulan Ma et al. This is an open access article distributed under the Creative Commons Attribution License, which permits unrestricted use, distribution, and reproduction in any medium, provided the original work is properly cited.

Metabolic energy preferentially produced by glycolysis was an advantageous metabolic phenotype of cancer cells. It is also an essential contributor to the progression of multidrug resistance in cancer cells. By developing human breast cancer MCF-7 cells resistant to doxorubicin (DOX) (MCF-7/MDR cells), the effects and mechanisms of 2-deoxy-D-glucose (2DG), a glucose analogue, on reversing multidrug resistance were investigated. 2DG significantly inhibited the viability of MCF-7/MDR cells and enhanced DOX-induced apoptosis by upregulating protein expression of AMPK $\alpha$, P53, and caspase- 3 . The study demonstrated that energy restriction induced by 2DG was relevant to the synergistic effect of 2DG and DOX. The proteins of multidrug gene (the MDR-related protein, MRP1) and P-glycoprotein (P-gp) in MCF-7/MDR cells were downregulated after exposure to 2DG, accompanied with the suppression of the activity of ATP-dependent drug-efflux pump and transmembrane transporter, increasing the intracellular accumulation of DOX to reverse the chemoresistance in multidrug cancer cells.

\section{Introduction}

Scientific literature indicates that cancer cells predominantly generate energy by a high rate of glycolysis rather than the Krebs cycle of mitochondrial metabolism even when oxygen is abundant, which is called Warburg's effect [1]. Glycolytic activity is an inefficient way for energy production. Elevated amount of glucose is consumed to meet the rapid progression of the tumor. Based on this, the abnormal uptake of 2$\left[{ }^{18} \mathrm{~F}\right]$ fluoro-2-deoxy-D-glucose (FDG) has been utilized in imaging by positron emission tomography/computed tomography (PET/CT) [2]. Furthermore, due to the accumulation of lactic acid from Warburg's effect, the acidification of extracellular microenvironment favors the progression and metastases of tumor via upregulation of metallo-proteinase and cysteine proteinase activity and secretion [3]. It has been suggested that the metabolic characteristic of cancer cells facilitates cancer cells' survival and contributes to their resistance to conventional cancer therapies, such as chemotherapy and radiotherapy [4].
Breast cancer is one of the common malignant tumors affecting adult women. Doxorubicin (DOX) has been considered as one of the potent first-line chemotherapeutic agents for breast cancer treatment due to its high efficacy and tolerance. However, the development of multidrug resistance (MDR) is an unfavorable factor in the poor prognosis for breast cancer. Meantime, the development of MDR is accompanied with the overexpression of ATP-dependent drug-efflux pump, such as p-glycoprotein (P-gp), multidrug resistance protein 1 (MRP1), and the breast cancer resistance protein (BCRP), which is thought to be an essential obstacle to reducing intracellular drug accumulation in tumor eradication [5].

In the current study, we developed DOX-resistant MCF7 cells and examined the antiproliferative effect of DOX when the cellular metabolism was modulated with a glucose inhibitor. The results showed that energy restriction sensitized the DOX-resistant MCF-7 cells and enhanced the apoptosis induced by DOX. Since the glucose inhibitor 
induced a dramatic reduction in cellular ATP levels, the possible mechanism may be related to the functions of drug-efflux pumps, where the activity of transmembrane transporters regulates the energy restriction.

\section{Materials and Methods}

2.1. Drugs. Doxorubicin (DOX) and 2-deoxy-D-glucose (2DG) were purchased from Sigma-Aldrich Co. (St. Louis, MO, USA). DOX was dissolved in water as a $1 \mathrm{M}$ stock solution. 2DG was dissolved in phosphate-buffered saline (PBS) to prepare a stock solution of $1 \mathrm{M}$. Drugs were serially diluted with culture medium before use.

2.2. Cell Line. Human breast carcinoma cell line MCF-7, obtained from Shanghai Institutes for Biological Science, Chinese Academy of Sciences (Shanghai, China), was maintained in Dulbecco's Modified Eagle Medium (HyClone, Beijing, China) containing glucose $(4.5 \mathrm{~g} / \mathrm{L})$ and supplemented with penicillin (100 units $/ \mathrm{mL})$, streptomycin $(100 \mu \mathrm{g} / \mathrm{mL})$, glutamate $(2 \mathrm{mM})$, and $10 \%$ fetal bovine serum (Gibco Invitrogen, Grand Island, NY, USA). MCF-7/MDR cells, resistant to DOX, were derived by stepwise selection with DOX and were maintained in the presence of $1 \mu \mathrm{M}$ DOX. Cells were grown in normal culture medium for 1 week before each experiment. Cells were maintained in a humidified atmosphere of $5 \% \mathrm{CO}_{2}$ in air at $37^{\circ} \mathrm{C}$.

2.3. Cell Viability Assay. The quantity of viable cells after treatment with various agents was determined with the Cell Counting Kit-8 (CCK8) (Dojindo, Shanghai, China) according to the manufacturer's protocol. Briefly, cells were plated into 96 -well plates $\left(100 \mu \mathrm{L}, 5 \times 10^{3} /\right.$ well $)$ and allowed to adhere overnight and were treated with various concentrations of drugs for $24 \mathrm{~h}$. The culture medium was replaced with fresh medium containing CCK8 solution $(10 \% \mathrm{~V} / \mathrm{V})$ and the plates were incubated for additional $4 \mathrm{~h}$ at $37^{\circ} \mathrm{C}$. The absorbance at $450 \mathrm{~nm}$ was measured on a microplate reader (Bio-Tek, USA). The cell viability was calculated by the formula

$$
\text { cell viability }(\%)=\frac{\left(\mathrm{OD}_{\text {treatment }}-\mathrm{OD}_{\text {blank }}\right)}{\left(\mathrm{OD}_{\text {control }}-\mathrm{OD}_{\text {blank }}\right)} \times 100 \% \text {. }
$$

2.4. Apoptosis Analysis. Culture medium containing floating cells was removed and retained. The monolayer cells were rinsed with PBS and harvested with trypsin (without EDTA). The cells were then pooled with the floating cells, washed, and resuspended in $100 \mu \mathrm{L}$ binding buffer supplemented with Annexin V-FITC $(5 \mu \mathrm{L})$ and PI $(5 \mu \mathrm{L})$. All samples were incubated in the dark room. The stain was stopped by adding $300 \mu \mathrm{L}$ binding buffer. Apoptotic cells were analyzed by a flow cytometer (Gallios, Beckman Coulter, USA). At least 10,000 cells from each sample were analyzed.

2.5. Determination of Intercellular ATP. Intracellular ATP was determined as previously described using a Bioluminescence Detection Kit for ATP (Promega Co., USA) [6].
Briefly, cells were treated with 2DG (from $5 \mathrm{mM}$ to $80 \mathrm{mM}$ ) for $24 \mathrm{~h}$. Whole-cell extracts of $1 \times 10^{5}$ cells were prepared in lysis buffer. Pellet debris was centrifuged briefly and $10 \mu \mathrm{L}$ of supernatant was mixed with $100 \mu \mathrm{L}$ Luciferase Assay Reagent of ATP assay to measure the light produced. Fluorescence light emission was measured with a microplate reader (BioTek, USA).

2.6. Estimation of $\mathrm{Na}^{+}, \mathrm{K}^{+}$-ATPase Activity. According to the method described previously [7], determination of $\mathrm{Na}^{+}-\mathrm{K}^{+}$ATPase activity was carried out with some modifications. Aliquots of cell extract were prepared by ultrasonication. Samples were added to an enzyme reaction mixture $(100 \mathrm{mM}$ Tris- $\mathrm{HCl}$ buffer, $125 \mathrm{mM} \mathrm{NaCl}, 75 \mathrm{mM} \mathrm{KCl,} 7.5 \mathrm{mM} \mathrm{MgCl}$, and $10 \mathrm{mM} \mathrm{Na}_{2} \mathrm{ATP}$, at $\mathrm{pH}$ 7.5). The reaction was incubated at $28^{\circ} \mathrm{C}$ for $20 \mathrm{~min}$ followed by immediate ice bath to stop the reaction. Released inorganic phosphate $(\mathrm{Pi})$ was measured at $650 \mathrm{~nm}$ in the color reagent (1\% Tween 20 and $1 \%$ ammonium molybdate in $0.9 \mathrm{M} \mathrm{H}_{2} \mathrm{SO}_{4}$ ).

2.7. Rhodamine 123 (Rh 123) Accumulation Study. The cellular accumulation of fluorescent dye Rh 123 was used to examine the effects of $2 \mathrm{DG}$ on the functional activity of P-gp according to the method of Fontaine et al. [8]. MCF-7/MDR cells at a density of $1 \times 10^{5}$ cells/mL in exponential growth were preincubated with various concentrations of $2 \mathrm{DG}$ or combined with $30 \mu \mathrm{M}$ DOX for $4 \mathrm{~h}$. And then, the cells were incubated in the presence of $10 \mu \mathrm{M}$ Rh123 at $37^{\circ} \mathrm{C}$ for up to $120 \mathrm{~min}$. After removing the dye, the fluorescence intensity of 10,000 cells was measured using flow cytometry.

2.8. Real-Time Polymerase Chain Reaction Analysis. Quantitative real-time reverse transcription polymerase chain reaction (qRT-PCR) was employed for gene expression analyses. Amplification of genes was performed using SYBR Green Real Master Mix (Tianjin, China). Sense and antisense primers used for amplification in this study were as follows: $5^{\prime}$-ATCGCCGTGTTTGGCTACTCC-3' and $5^{\prime}$ AAGCGGTTCACCAGGTTCCC-3' for MRP1; $5^{\prime}$-GGGTGGTGTCACAGGAAGAGATT- $3^{\prime}$ and $5^{\prime}$-GGCTGTCTAACAAGGGCACGA-3' for P-gp; and $5^{\prime}$-GGAGTCCACTGGCGTCTTC-3' and $5^{\prime}$-GCTGATGATCTTGAGGCTGTTG-3' for GAPDH. All real-time experiments were run in triplicate and a mean value was used for the determination of mRNA levels. Relative mRNA expression levels for MRP1, $\mathrm{P}$-gp, and GAPDH were determined using the $2^{-\Delta \Delta C t}$ method and normalized to the GAPDH.

2.9. Western Blot Analysis. After treatment, the cell lysate was prepared with RIPA lysis buffer. The protein content of the extracts was determined using BCA protein assay kit (Beyotime, China). An equal amount of total protein was subjected to $10 \%$ SDS-PAGE and transferred to PVDF membrane (Millipore, Bedford, USA). The following antibodies were used to probe the corresponding proteins: anti-phosphoAMPK $\alpha$ (CST, USA), anti-AMPK $\alpha$ (CST, USA), caspase-3 (Epitomics, USA), anti-p53 (Epitomics, USA), anti-phosphop53 (Epitomics, USA), and anti-GAPDH (Beyotime, China). 

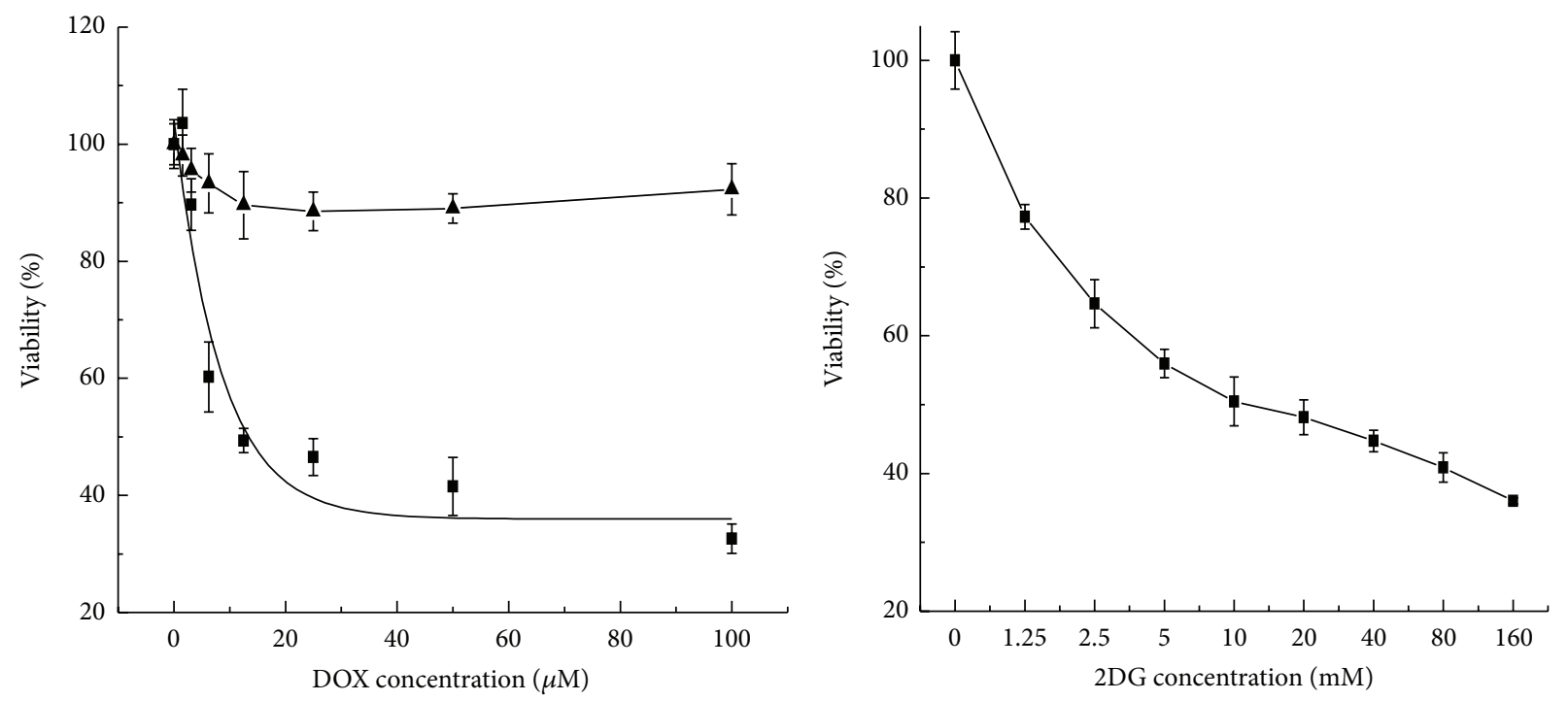

- MCF-7

-4- MCF-7/MDR

(a)

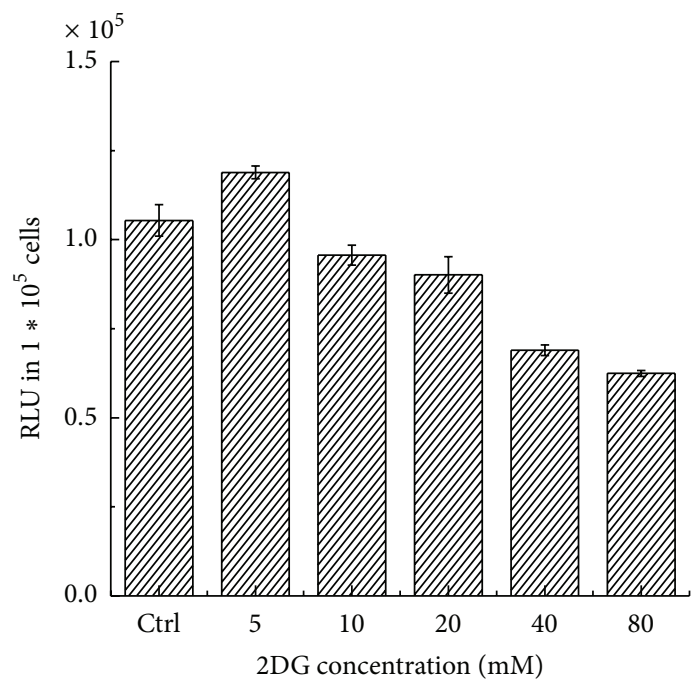

(c)

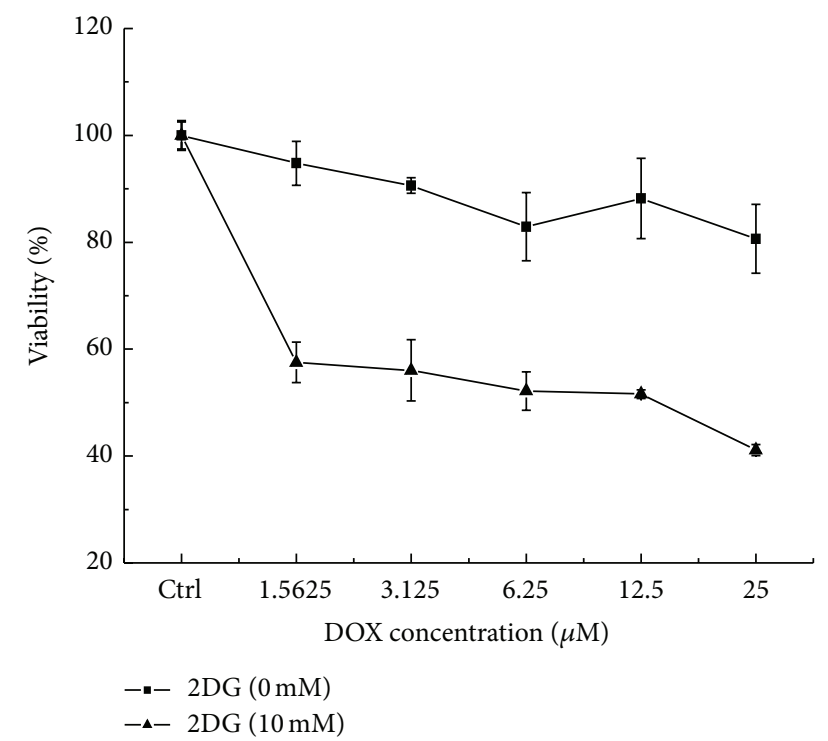

(d)

FIGURE 1: Energy restriction induced by 2DG inhibited the proliferation of MCF-7/MDR cells. (a) The establishment of MCF-7/MDR cells. (b) 2DG depressed the proliferation of MCF-7/MDR cells in a dose-dependent manner. (c) 2DG depleted the level of intercellular ATP. (d) 2DG enhanced the cytotoxicity of DOX.

GAPDH was used for the loading control. The protein bands were visualized using the ChemiDoc XRS system (Bio-Rad Laboratories, USA). The protein level was determined with Quantity One software (Bio-Rad Laboratories).

2.10. Statistics. Experimental data were presented as mean with standard deviation for at least three independent experiments and analyzed with the SPSS 13.0 software. The difference between groups was assessed using Student's $t$-test and $P<0.05$ was considered to be significant.

\section{Results}

3.1. 2DG Inhibited the Proliferation of DOX-Resistant Breast Cancer Cells by Depleting Intracellular ATP Supplement. The resistance of MCF-7/MDR cell line towards DOX was testified with CCK8 assay. The results revealed that $\mathrm{IC}_{50}$ values of DOX for MCF-7 and MCF-7/MDR cells were $23.52 \mu \mathrm{M}$ and $678.15 \mu \mathrm{M}$, respectively (Figure 1(a)). It confirmed that MCF-7/MDR was 300-fold more resistant to the effects of DOX, compared to MCF-7 cells. Furthermore, MCF-7/MDR cells exhibited obvious growth depression towards 2DG in a 
concentration dependent manner (Figure 1(b)). To examine whether the effect is attributed to the lack of cellular ATP, intracellular ATP levels were detected when MCF-7/MDR cells were exposed to $2 \mathrm{DG}$ at various concentrations for $24 \mathrm{~h}$. Except that $5 \mathrm{mM} 2 \mathrm{DG}$ stimulated the generation of cellular ATP, a dose-dependent decrease in the ATP level was found from $10 \mathrm{mM}$ to $80 \mathrm{mM}$ (Figure $1(\mathrm{c})$ ). To verify the interaction between DOX and 2DG, $10 \mathrm{mM}$ 2DG was added to sensitize MCF-7/MDR cells to DOX. The results showed that $\mathrm{DOX} \mathrm{IC}_{50}$ in the presence of $10 \mathrm{mM} 2 \mathrm{DG}$ declined to $1.56 \mu \mathrm{M}$, which led to a more than 400 -fold increase of DOX cytotoxicity (Figure 1(d)). It was revealed that energy restriction sensitized MCF-7/MDR cells towards DOX.

\subsection{Intracellular Energy Restriction Reversed the Resistance of} Cells towards DOX by Depressing Drug-Efflux Transporters. The occurrence of multidrug resistance has been widely recognized as the overexpression of efflux transporters, such as P-glycoprotein (P-gp) and the MDR-related protein (MRP1). To determine whether changes in the mRNA levels of these genes correlate with the effect of 2DG in MCF-7/MDR cells, real-time PCR was carried out to evaluate the differences in expression level of the chosen genes. As shown in Figure 2(a), pretreatment MCF-7/MDR cells with $20 \mathrm{mM}$ 2DG decreased MDR1 expression by $23 \pm 0.1 \%$ and P-gp expression by $79 \pm$ $1.5 \%$, compared to the control group. In parallel, we measured the cellular accumulation of Rd 123 to evaluate the function of P-gp (Figure 2(b)). As expected, 2DG treatment significantly increased Rd 123 accumulation. The intensity of Rd 123 fluorescence was remarkably increased upon increasing the concentration of $2 \mathrm{DG}$ from $64.66 \pm 3.37 \%$ at $5 \mathrm{mM}$ to $108.94 \pm$ $1.82 \%$ at $20 \mathrm{mM}$.

In this study, we also examined the activity of cellular $\mathrm{Na}^{+}-\mathrm{K}^{+}$-ATPase after the MCF-7/MDR cells were exposed to 2DG. As shown in Figure 2(c), the independent administration of $30 \mu \mathrm{M}$ DOX activated the $\mathrm{Na}^{+}-\mathrm{K}^{+}$-ATPase significantly. However, the addition of $2 \mathrm{DG}$ depressed its activity up to $50 \%$ at the higher treatment concentration examined $(10 \mathrm{mM})$ compared with the control group, and the inhibition was up to $70 \%$ in the presence of $30 \mu \mathrm{M}$ DOX.

\subsection{Energy Depletion Enhanced the Cytotoxicity of Doxoru-} bicin by Inducing Cell Apoptosis. To verify the chemosensitized enhancement, the effect of 2DG on the cytotoxicity of DOX was investigated. We found that the $2 \mathrm{DG}$ was effective in enhancing the growth inhibition of $30 \mu \mathrm{M}$ DOX against MCF-7/MDR cells at even suboptimal concentration ( $5 \mathrm{mM}$ ) (Figure 3(a)). Meanwhile, the floating and adherent cells were harvested and stained with Annexin V-FITC for the analyses of apoptosis using flow cytometer. The apoptotic cell population comprised early apoptotic cells and those in the late stages. The results showed that the percentage of apoptotic cells induced by DOX was remarkably enhanced with the concentrations of $2 \mathrm{DG}$. The percentage was almost increased by 3 -fold from $6.02 \pm 0.87 \%$ at single administration of $30 \mu \mathrm{M}$ DOX to $16.26 \pm 0.39 \%$ at the combination of $20 \mathrm{mM}$ $2 \mathrm{DG}$ and $30 \mu \mathrm{M}$ DOX (Figure 3(b)). To further explore the signal regulatory pathways, the protein levels of AMPK $\alpha, \mathrm{p} 53$, and caspase- 3 were analyzed by western blotting in total cell lysates and the result was shown in Figure 3(c). AMPK $\alpha$ had a very low expression in nontreated cells, but it would be accumulated in cells as cells were exposed to either 2DG or combination with DOX. In particular, $10 \mathrm{mM}$ 2DG induced the significant activation of phospho-AMPK $\alpha$ no matter if cells received the administration of DOX or not. In addition, the level of phospho-p53 was increased by 2 -fold and 1.89 -fold in the combination group compared to those in response to DOX or 2DG single treatment group, respectively. Caspase3 was a member of the cysteine-aspartic acid protease family and acted as an essential executive enzyme in the apoptosis signal pathway. As expected, its expression was increased after treatment with DOX. This increased activity was further augmented by addition of 2DG to reach a 1.13-fold expression, compared to DOX-treated cells. The finding was in concert with our previous apoptosis analysis and indicated that the combination of $2 \mathrm{DG}$ could further promote MCF-7/MDR cells apoptosis.

\section{Discussion}

Energy metabolism is the foundation of cell growth, proliferation, and differentiation. Cell proliferation relies on its demand of energy supply, particularly the status of intracellular ATP. The alteration of normal biochemical processes of glucose in cancer not only compensates the lack of growth nutrition factors, but also favors the tumor in the buildup of biomass more rapidly [9]. In human glioblastoma multiforme, the Warburg effect is crucial and correlated with worse overall survival of patients [10]. At present, targeting cancer cell metabolism provides a new promising strategy to preferentially kill the malignant cells.

2DG is a widely studied glucose analogue, which inhibits glucose metabolism by competitively inhibiting the uptake and utilization of glucose. 2DG is phosphorylated to generate $2 \mathrm{DG}-\mathrm{PO}_{4}$ during the digestion. The latter is trapped in the cells and stops to be further metabolized to fulfill the inhibition of the glucose metabolism [11]. In our experiment, 2DG was used to inhibit the generation of ATP. The results presented that 2DG depleted the level of intercellular ATP and the generation of ATP was progressively lower with the increase in 2DG concentrations. Besides, AMPK $\alpha$ was activated and phosphorylated after cells were exposed to 2DG. AMPK $\alpha$ is a prominent sensor of cellular energy status, which acts as a marker of the level of intercellular ATP pool. 2DG decreased the level of ATP and increased the cellular AMP/ATP ratio, which inevitably led to the activation of AMPK $\alpha$ to promote catabolic processes and inhibit anabolic processes in response to ATP cellular demands [12]. Equally, we detected the depression of cell growth. It was postulated that 2DG affected the capacity of cell proliferation by depressing intercellular ATP.

Multidrug resistance is a phenomenon when cells acquire simultaneous resistance to certain chemotherapeutic agents, which frequently contribute to the overexpression of drugefflux pump to reduce intracellular drug accumulation [13]. P-Glycoprotein (P-gp), multidrug resistance protein (MRP), and breast cancer resistance protein (BCRP), which are called the ATP-binding cassette $(\mathrm{ABC})$ transporters, are a 


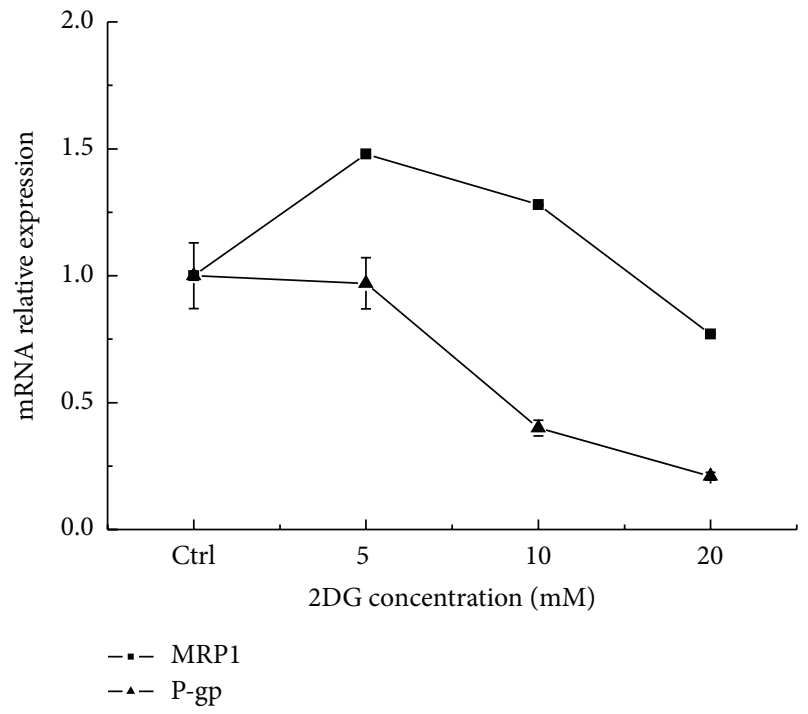

(a)
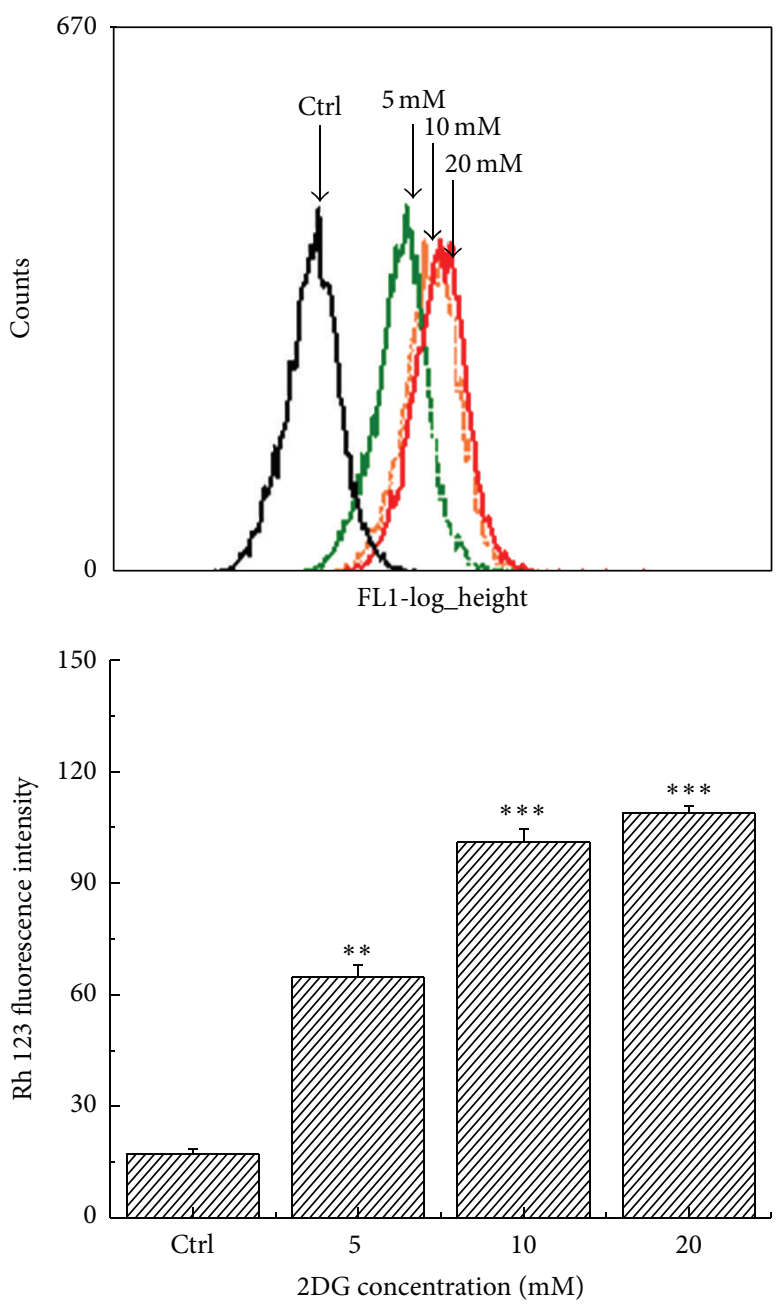

(b)

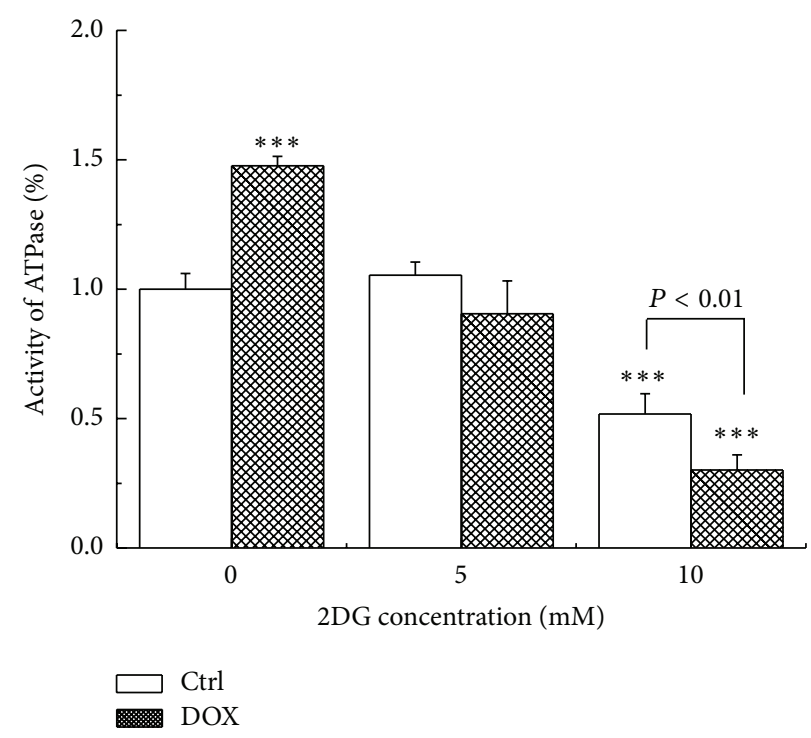

(c)

FIGURE 2: Energy restriction induced by 2DG depressed the activity of transmembrane transporter system. (a) 2DG decreased the mRNA expression of multidrug gene, MRP1, and P-gp. (b) The intensity of Rh 123 fluorescence was detected with flow cytometer. ${ }^{* *} P<0.01$; ${ }^{* * *} P<0.001$ versus control group. (c) The activity of cellular $\mathrm{Na}^{+}-\mathrm{K}^{+}$-ATPase was examined. ${ }^{* * *} P<0.001$ versus control group. 


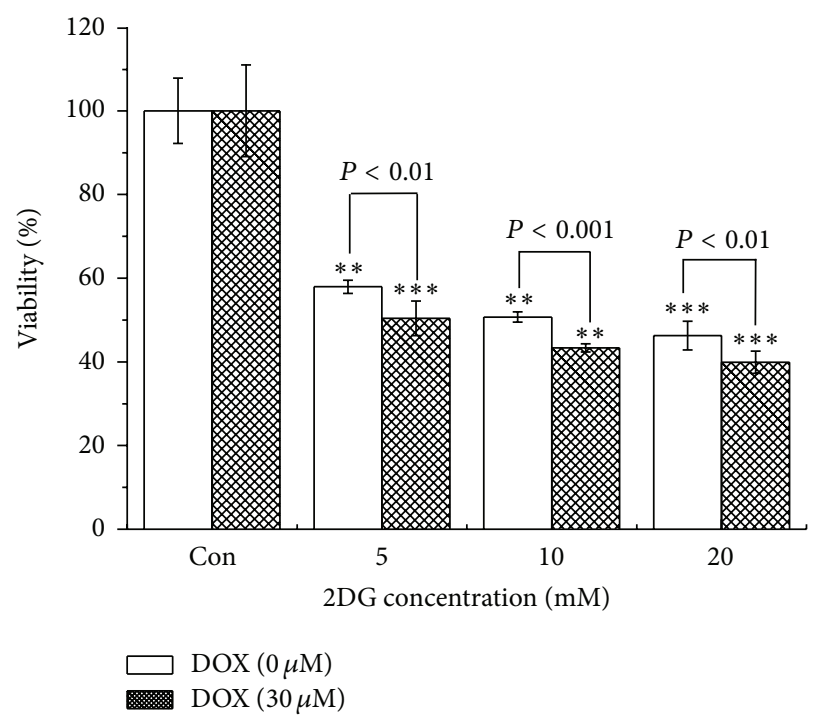

(a)

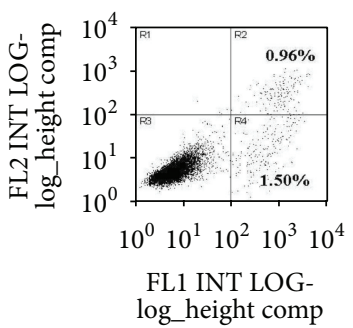

(A)

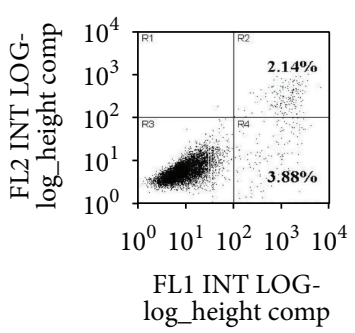

(D)

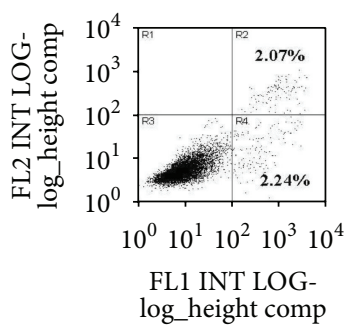

(B)

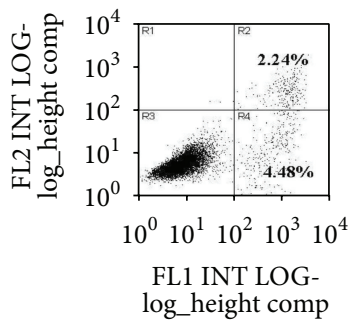

(E)

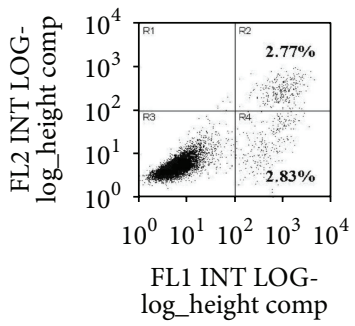

(C)

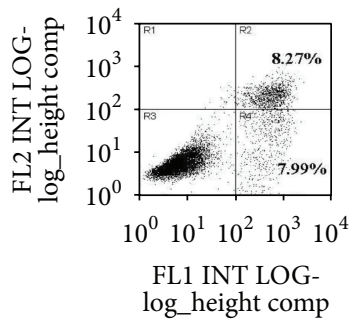

(F)
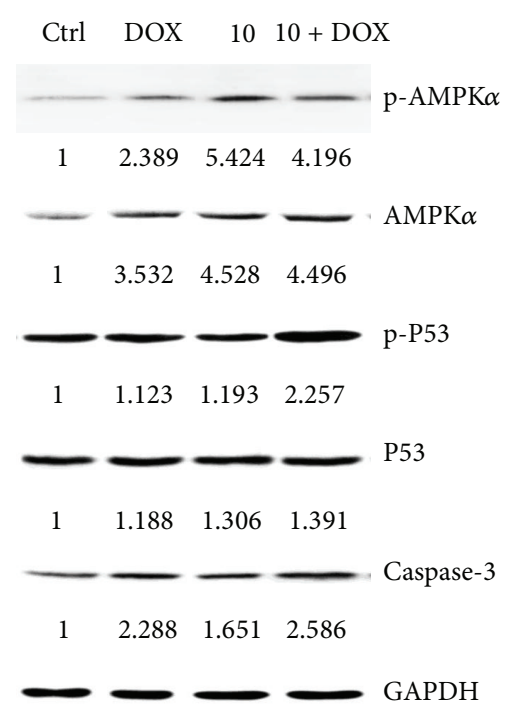

(c)

(b)

FIGURE 3: 2DG sensitized MCF-7/MDR cells towards DOX. (a) The cytotoxicity of DOX was enhanced with the increase of 2DG concentration. ${ }^{* *} P<0.01 ;{ }^{* * *} P<0.001$ versus control group. (b) Cell apoptosis was increased under the combination of $2 \mathrm{DG}$ and DOX. (A) The control group; (B) $10 \mathrm{mM} 2 \mathrm{DG}$ group; (C) $20 \mathrm{mM}$ 2DG group; (D) $30 \mu \mathrm{M}$ DOX; (E) the combination of $10 \mathrm{mM} 2 \mathrm{DG}$ and $30 \mu \mathrm{M}$ DOX; (F) the combination of $20 \mathrm{mM} 2 \mathrm{DG}$ and $30 \mu \mathrm{M}$ DOX. (c) The western blot assay was carried out after being exposed to either 2DG (10 mM) or DOX $(30 \mu \mathrm{M})$ or the combination of $2 \mathrm{DG}$ and DOX.

group of plasma membrane proteins that are associated with the development of drug resistance. Previous observations reported that the metabolic depletion was also accompanied by the potentiation of the depression of the drug-efflux and drug-sequestration systems [14]. Could the ATP depletion induced by $2 \mathrm{DG}$ chemosensitize and potentiate cytotoxic effect of DOX in MDR cells? The results showed that the drugefflux systems were ATP-dependent, and a substantial mRNA decrease of P-gp and MRP1 was highly responsive to 2DG treatment. The less prominent activation of transmembrane transport system could retain the accumulation of DOX in cells, as seen in the $\mathrm{Rh} 123$ accumulation assay. It is suggested that the inhibition on sensitization of DOX-resistant cell by P-gp and MRP1 was the result of 2DG-induced ATP depletion. Furthermore, the maintenance of $\mathrm{pH}$ gradients across organelle membranes was also essential for drug sequestration in MDR cells when the transportation was monitored by the activity of ATP-dependent pump [15]. It was observed that $2 \mathrm{DG}(10 \mu \mathrm{M})$ significantly impaired the activity of $\mathrm{Na}^{+}-\mathrm{K}^{+}$-ATPase in MCF-7/MDR cells. $\mathrm{Na}^{+}-\mathrm{K}^{+}$ATPase acts as a signal transducer/integrator to maintain resting potential, participating in transport and regulating 
cell energy expenditure. It provides the sodium gradient to import glucose, amino acids, and other nutrients into cells. For neurons, $\mathrm{Na}^{+}-\mathrm{K}^{+}$-ATPase is responsible for up to $2 / 3$ of the cell's energy consumption [16]. When glucose metabolism is inhibited, the reduction in $\mathrm{Na}^{+}-\mathrm{K}^{+}$-ATPase activity is inevitable. In turn, the reduction in intracellular ATP levels might be potentiated by the repression of the levels of membrane transport system.

However, the depression of the activity of transmembrane transporters by 2DG was probably not the only mechanism involved in reversing the resistance of MCF-7/MDR cells to DOX. DOX is a feasible first-line chemotherapeutic agent, which induces DNA strand breakages by inhibiting the enzyme topoisomerase II. When DNA damage occurs, a large amount of ATP is required to form a dynamic repair complex at double-strand break sites [17]. In our previous studies, depletion of intracellular ATP retarded the repair of the potential DNA lethal damage [6]. It was suggested that 2DG depleting ATP pool and enhancing DNA damage might be a potential mechanism of the decreased DOX-induced cell viability by regulating the energy metabolism.

In conclusion, we propose the fact that $2 \mathrm{DG}$ reversed the resistance of MCF-7/MDR cells and enhanced DOX-induced apoptosis by interfering with the biochemical metabolism of glucose. The process was related to the depletion of intracellular ATP level, the inactivation of drug-efflux pump, and the depression of transmembrane transporters. Understanding energy metabolism in a combination of chemotherapeutic agents will provide the basis for rational suggestions of coadministration of energy metabolism inhibitor to maximize the killing of multidrug resistant cells.

\section{Conflict of Interests}

The authors declare that there is no conflict of interests regarding the publication of this paper.

\section{Authors' Contribution}

Shulan Ma and Rongfei Jia contributed equally to this work.

\section{Acknowledgments}

The authors gratefully acknowledge the number of researchers who have contributed to this work. The authors thank Zemin Wang for kind comments, Kai Sun and Kevin Hopkins for critical reading of the paper, and Chunlin Shao for facilities provision. This work was financially supported by the National Natural Science Foundation (nos. 11105031, 81102668), the National Key Laboratory Opening Funds (SKL201410SIC), and the Youth Scientific Research Foundation of Fudan University of China (2014).

\section{References}

[1] G. Kroemer and J. Pouyssegur, "Tumor cell metabolism: cancer's Achilles' heel," Cancer Cell, vol. 13, no. 6, pp. 472-482, 2008.
[2] S. S. Gambhir, "Molecular imaging of cancer with positron emission tomography," Nature Reviews Cancer, vol. 2, no. 9, pp. 683-693, 2002.

[3] R. van Horssen, R. Buccione, M. Willemse, S. Cingir, B. Wieringa, and F. Attanasio, "Cancer cell metabolism regulates extracellular matrix degradation by invadopodia," European Journal of Cell Biology, vol. 92, no. 3, pp. 113-121, 2013.

[4] U. E. Martinez-Outschoorn, Z. Lin, Y.-H. Ko et al., "Understanding the metabolic basis of drug resistance: therapeutic induction of the Warburg effect kills cancer cells," Cell Cycle, vol. 10, no. 15, pp. 2521-2528, 2011.

[5] A. A. Kovalev, D. A. Tsvetaeva, and T. V. Grudinskaja, "Role of ABC-cassette transporters (MDR1, MRP1, BCRP) in the development of primary and acquired multiple drug resistance in patients with early and metastatic breast cancer," Experimental Oncology, vol. 35, no. 4, pp. 287-290, 2013.

[6] B. Shen, P.-J. He, and C.-L. Shao, "Norcantharidin induced DU145 cell apoptosis through ROS-mediated mitochondrial dysfunction and energy depletion," PLOS ONE, vol. 8, no. 12, Article ID e84610, 2013.

[7] C.-H. Tang, W.-Y. Wu, S.-C. Tsai, T. Yoshinaga, and T.-H. Lee, "Elevated $\mathrm{Na}^{+} / \mathrm{K}^{+}$-ATPase responses and its potential role in triggering ion reabsorption in kidneys for homeostasis of marine euryhaline milkfish (Chanos chanos) when acclimated to hypotonic fresh water," Journal of Comparative Physiology B: Biochemical, Systemic, and Environmental Physiology, vol. 180, no. 6, pp. 813-824, 2010.

[8] M. Fontaine, W. F. Elmquist, and D. W. Miller, "Use of rhodamine 123 to examine the functional activity of P-glycoprotein in primary cultured brain microvessel endothelial cell monolayers," Life Sciences, vol. 59, no. 18, pp. 1521-1531, 1996.

[9] M. G. V. Heiden, L. C. Cantley, and C. B. Thompson, "Understanding the warburg effect: the metabolic requirements of cell proliferation," Science, vol. 324, no. 5930, pp. 1029-1033, 2009.

[10] A. Wolf, S. Agnihotri, J. Micallef et al., "Hexokinase 2 is a key mediator of aerobic glycolysis and promotes tumor growth in human glioblastoma multiforme," Journal of Experimental Medicine, vol. 208, no. 2, pp. 313-326, 2011.

[11] Z. Zhu, W. Jiang, J. N. McGinley, and H. J. Thompson, "2deoxyglucose as an energy restriction mimetic agent: effects on mammary carcinogenesis and on mammary tumor cell growth in vitro," Cancer Research, vol. 65, no. 15, pp. 7023-7030, 2005.

[12] B. Viollet, S. Horman, J. Leclerc et al., "AMPK inhibition in health and disease," Critical Reviews in Biochemistry and Molecular Biology, vol. 45, no. 4, pp. 276-295, 2010.

[13] O. Legrand, G. Simonin, A. Beauchamp-Nicoud, R. Zittoun, and J.-P. Marie, "Simultaneous activity of MRP1 and Pgp is correlated with in vitro resistance to daunorubicin and with in vivo resistance in adult acute myeloid leukemia," Blood, vol. 94, no. 3, pp. 1046-1056, 1999.

[14] A. V. Kabanov, E. V. Batrakova, and V. Y. Alakhov, "An essential relationship between ATP depletion and chemosensitizing activity of Pluronic block copolymers," Journal of Controlled Release, vol. 91, no. 1-2, pp. 75-83, 2003.

[15] C. Daniel, C. Bell, C. Burton, S. Harguindey, S. J. Reshkin, and C. Rauch, "The role of proton dynamics in the development and maintenance of multidrug resistance in cancer," Biochimica et Biophysica Acta, vol. 1832, no. 5, pp. 606-617, 2013.

[16] C. Howarth, P. Gleeson, and D. Attwell, "Updated energy budgets for neural computation in the neocortex and cerebellum," 
Journal of Cerebral Blood Flow and Metabolism, vol. 32, no. 7, pp. 1222-1232, 2012.

[17] R. A. Deshpande, G. J. Williams, O. Limbo et al., "ATP-driven Rad50 conformations regulate DNA tethering, end resection, and ATM checkpoint signaling," The EMBO Journal, vol. 33, no. 5, pp. 482-500, 2014. 


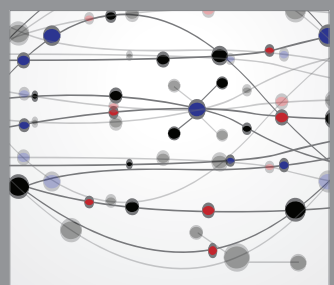

The Scientific World Journal
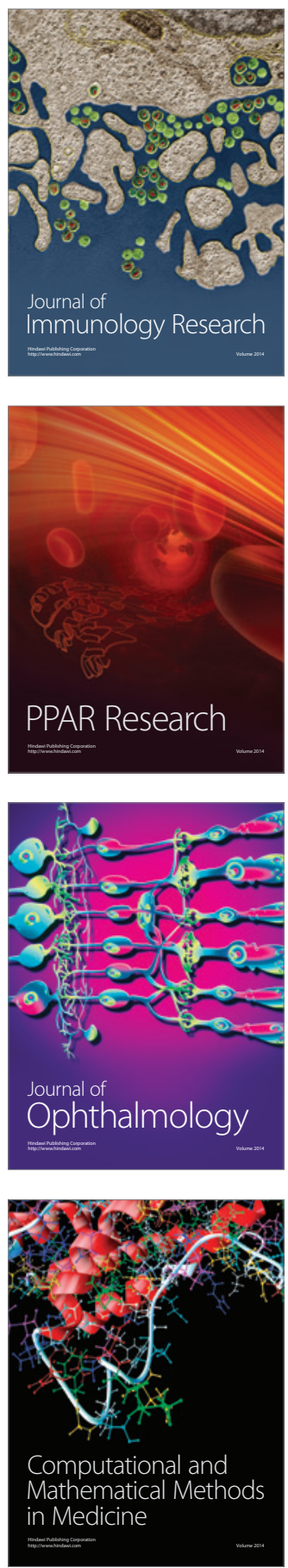

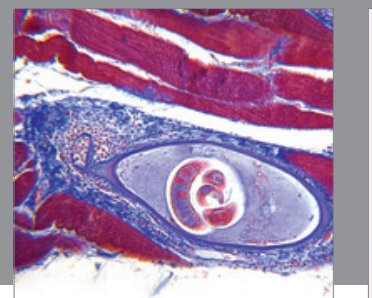

Gastroenterology

Research and Practice
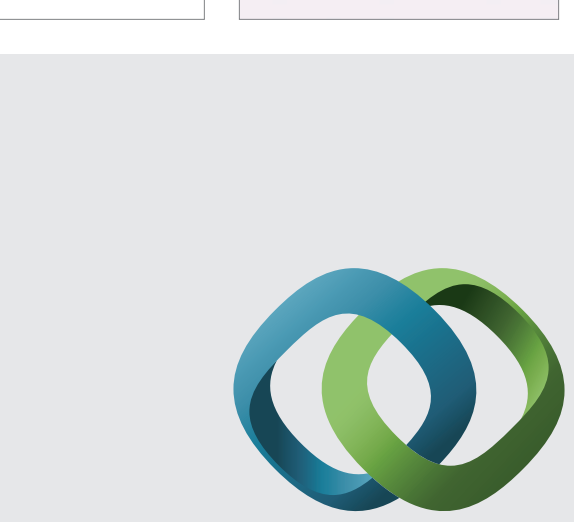

\section{Hindawi}

Submit your manuscripts at

http://www.hindawi.com
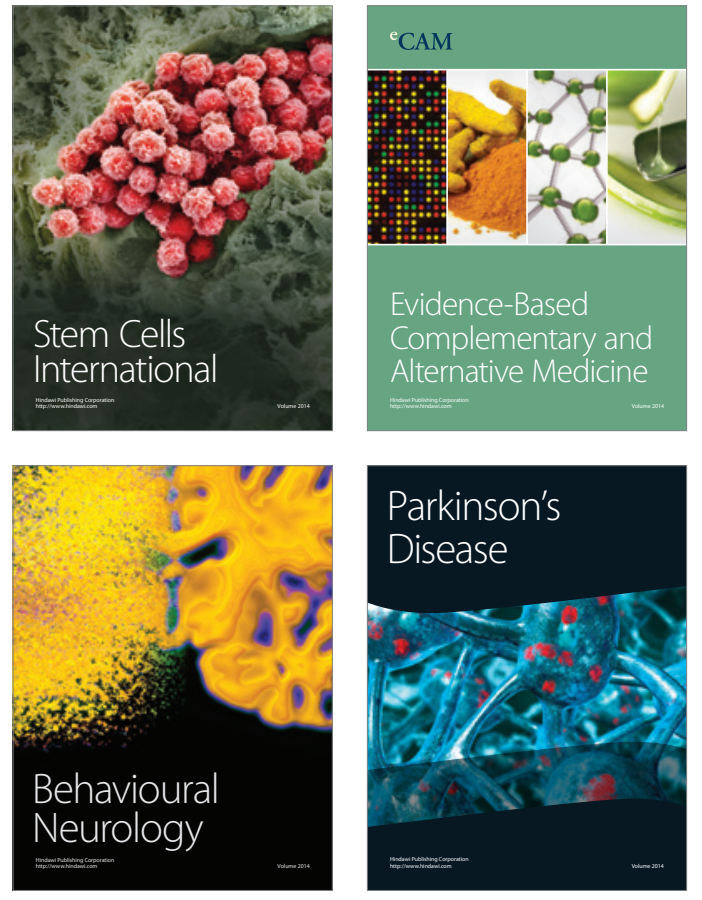
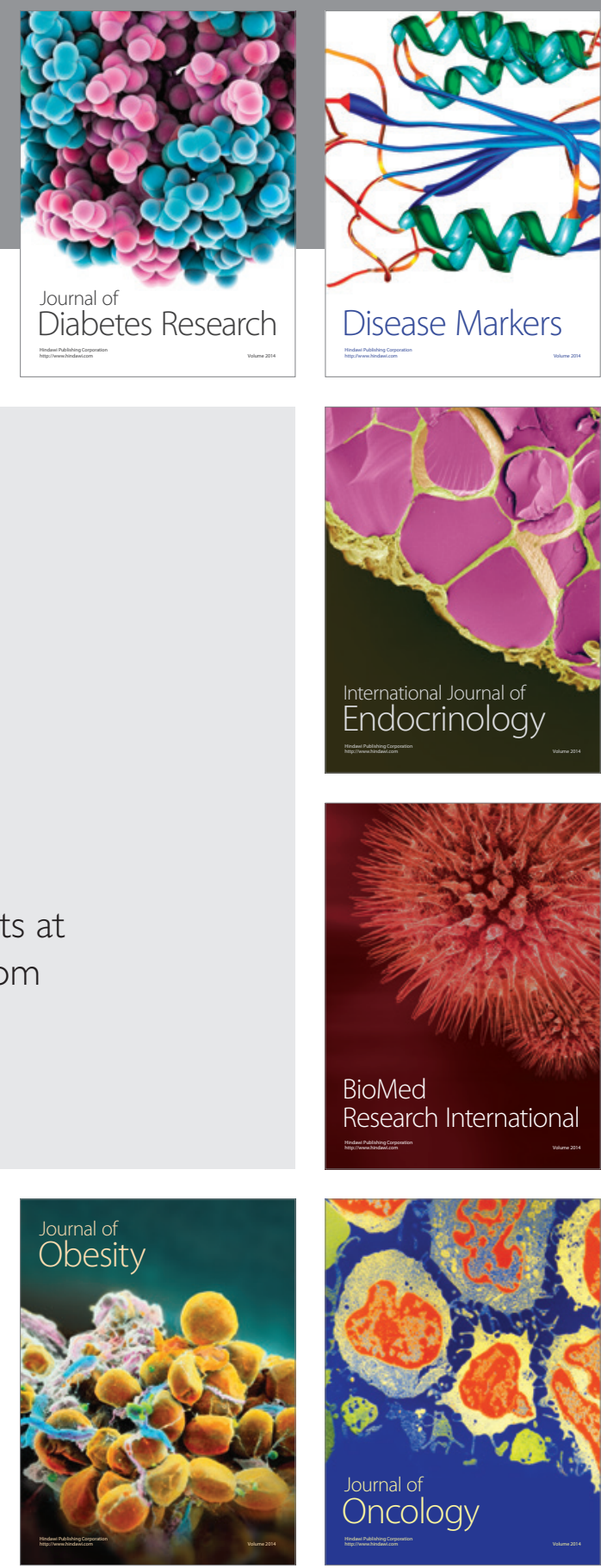

Disease Markers
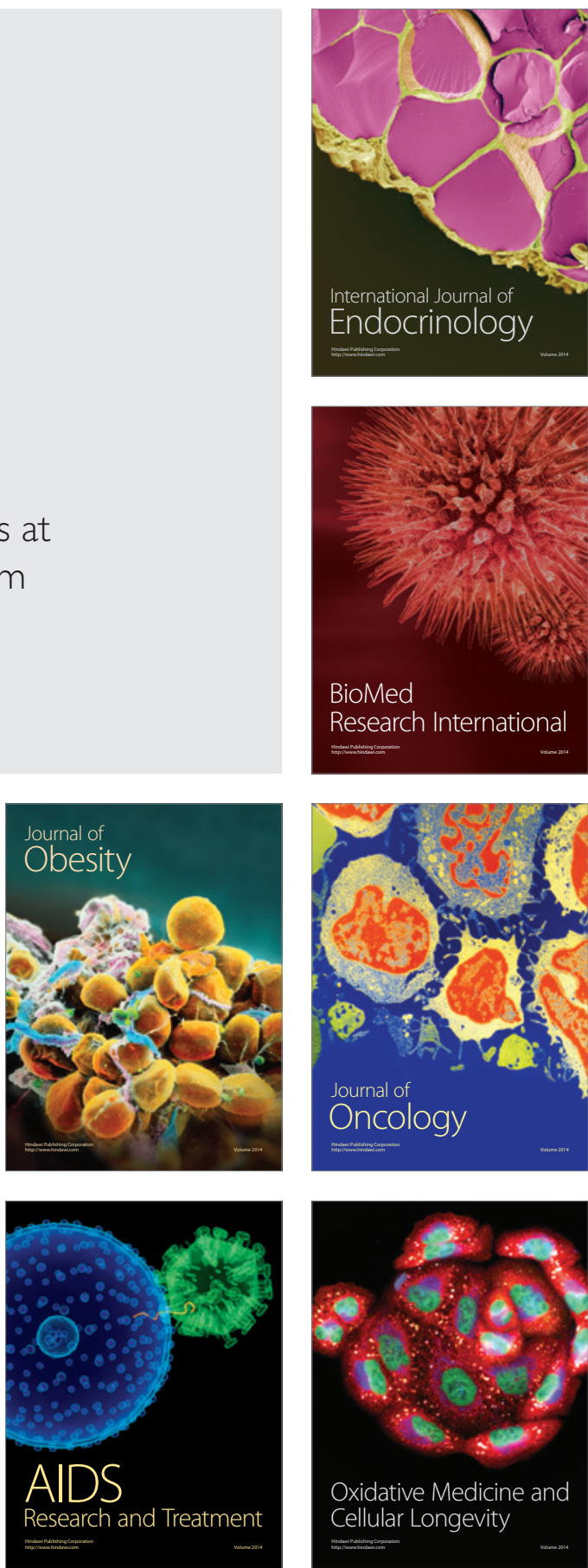